\title{
The Efficacy and Safety of Preoperative Lumbar Drain Placement in Anterior Skull Base Surgery
}

\author{
Paul D. Ackerman ${ }^{1}$ Drew A. Spencer ${ }^{1} \quad$ Vikram C. Prabhu ${ }^{1}$ \\ ${ }^{1}$ Department of Neurological Surgery, Loyola University Medical \\ Center, Maywood, Illinois, United States \\ Address for correspondence Vikram C. Prabhu, MD, FAANS, FACS, \\ Department Neurological Surgery, Loyola University Medical Center, \\ J Neurol Surg Rep 2013;74:1-9. \\ 2160 South First Avenue, Maywood, IL 60153, United States \\ (e-mail: vprabhu@lumc.edu).
}

\begin{abstract}
Keywords

- cerebrospinal fluid

- lumbar drain

- craniotomy

- anterior skull base

- endoscopic

This study assesses the efficacy of preoperative lumbar drain (LD) placement prior to elective open cranial and endoscopic anterior skull base (ASB) surgery in reducing postoperative cerebrospinal fluid (CSF) leak. A retrospective review of 93 patients who underwent LD placement at our institution between 2006 and 2011 was performed. Of these patients, 43 underwent elective LD placement prior to ASB surgery; 2 patients had evidence of CSF rhinorrhea prior to surgery, and 41 had no evidence of a preoperative CSF leak. Of those 41 patients, 2 developed CSF rhinorrhea $(2 / 41=4.9 \%)$ as a result of surgery-all in our endoscopic patient population $(N=21 ; 2 / 21=9.5 \%)$. No postoperative CSF leaks were noted in our open ASB surgery cohort $(N=20)$. Other complications were rare, but we encountered two instances of delayed malignant cerebral edema in the open ASB cohort that are discussed in detail. Overall, preoperative LD placement was found to be an effective means of preventing postoperative CSF leaks after ASB approaches, but potential and significant intracranial complications may occur in select patients that merit careful consideration prior to LD placement.
\end{abstract}

\section{Introduction}

Cerebrospinal fluid (CSF) leaks complicate operations of the cranial base, particularly those involving the anterior skull base (ASB). Kassam et al reported on their experience with almost 800 ASB procedures and reported a postoperative CSF leak, the most common complication they encountered, in $15.9 \%$ of patients. ${ }^{1}$ All cases of postoperative CSF leak resolved with placement of a lumbar drain (LD) supplemented at times by an endoscopic repair and, in one instance, with open cranial surgery. Other authors have reported a similar salutary effect of LD placement, and it has become a widely accepted strategy in managing postoperative CSF leaks. ${ }^{2-16}$

Lumbar drains are at times inserted prior to surgery. For open cranial ASB procedures, the presumed benefit of an LD is to decrease brain swelling and improve access to the tumor or floor of the anterior cranial fossa. In addition, it allows controlled drainage of CSF postoperatively, which is purported to reduce intracranial pressure and thereby facilitate adequate healing of the dural repair. For endoscopic ASB procedures, the LD allows delivery of positive pressure via injection of preservative-free saline to facilitate delivery of tumor from the suprasellar compartment, allow injection of fluorescein to detect occult CSF leaks, and facilitate healing of dural defect repair techniques.

However, LD placement prior to elective ASB surgery remains controversial. Lee et al reported the effectiveness of LD placement along with endoscopic repair as an effective strategy for CSF rhinorrhea related to defects of the frontal and sphenoid sinuses that are associated with meningocele or encephalocele. ${ }^{17}$ On the other hand, Casiano and Jassir questioned the utility of routine LD placement for the management of CSF rhinorrhea. ${ }^{18}$ In addition, other authors caution about the risk of neurological injury with the use of LD in ASB procedures. ${ }^{19}$ This paper presents our institution's experience with preoperative LD placement prior to open and endoscopic ASB surgery and evaluates the efficacy and safety of this strategy in preventing postoperative CSF leak. received

May 11, 2012

accepted

August 24, 2012

published online

January 2, 2013 (c) 2013 Georg Thieme Verlag KG Stuttgart · New York
DOI http://dx.doi.org/ 10.1055/s-0032-1331022. ISSN 2193-6358. 
Table 1 Indications for lumbar drain placement

\begin{tabular}{|l|l|}
\hline Indication for LD & $\begin{array}{l}\text { Number of } \\
\text { Patients }\end{array}$ \\
\hline ACF tumor resection & 43 \\
\hline $\begin{array}{c}\text { Patients with evidence } \\
\text { of preoperative CSF leak }\end{array}$ & 2 \\
\hline Encephalocele repair & 21 \\
\hline Traumatic or postoperative CSF leak & 13 \\
\hline $\begin{array}{c}\text { Aneurysm clipping or non-ACF tumor } \\
\text { resection }\end{array}$ & 9 \\
\hline LD trial (NPH) & 7 \\
\hline
\end{tabular}

Abbreviations: ACF, anterior cranial fossa; CSF, cerebrospinal fluid; LD, lumbar drain; NPH, normal pressure hydrocephalus.

\section{Methods}

We performed a retrospective chart review of all patients in the past 5 years who underwent LD placement at our institution to identify patients without evidence of a preoperative CSF leak who had a LD placed prior to elective ASB surgery. Since 2006, 93 patients had LDs placed for a variety of indications: to facilitate anterior cranial fossa (ACF) tumor resection by open $(N=20)$ or endoscopic $(N=23)$ approaches; prior to encephalocele repair for injection of fluorescein $(N=21)$, for traumatic CSF leaks $(N=13)$; before craniotomy for aneurysm clipping $(N=9)$; or as part of an LD trial related to normal pressure hydrocephalus $(N=7)$ (-Table 1). - Table 2 delineates the incidence of ASB tumors by pathology. We also analyzed whether there was any correlation between smoking, hypertension, or diabetes and the incidence of postoperative CSF leak. Of the 93 patients, 43 underwent elective LD placement prior to open $(N=20)$ or endoscopic $(N=23)$ ASB surgery. Of these 43 patients, 2 had evidence of CSF rhinorrhea prior to elective tumor resection; in both cases the LD and subsequent surgical repair was effective in eliminating a postoperative CSF leak. The remaining 41 patients were analyzed further.

The technique of LD placement is well described. ${ }^{13}$ Briefly, following intubation, with the patient in the lateral decubitus position, a catheter is inserted into the lumbar subarachnoid cistern at the L4-5 interlaminar space using a 14-gauge Tuohy needle. Cefazolin (Ancef; GlaxoSmithKline plc, London, United Kingdom) is administered prior to insertion and continued until the drain is removed. The LD is connected to a closed

Table 2 Primary pathology diagnosis

\begin{tabular}{|l|l|}
\hline Tumor Pathology & Incidence $(\boldsymbol{n}=\mathbf{4 3})$ \\
\hline Pituitary adenoma & 21 \\
\hline Meningioma & 10 \\
\hline Esthesioneuroblastoma & 9 \\
\hline Craniopharyngioma & 2 \\
\hline Pilocytic astrocytoma & 1 \\
\hline
\end{tabular}

system drainage bag, and CSF is drained in 10-cc aliquots during open cranial procedures to facilitate brain relaxation (up to a maximum of 30 to $50 \mathrm{cc}$ ). For endoscopic procedures, a 10 -cc aliquot of preservative-free saline is injected to increase pressure in the subarachnoid space and facilitate delivery of tumor from the suprasellar compartment. At times, $0.1 \mathrm{cc}$ of fluorescein (Fluorescite, Alcon Laboratories, Inc., Fort Worth, Texas, USA) diluted in 9.9 cc of preservativefree saline is injected through the LD to identify areas of dural dehiscence and CSF leaks.

Following surgery, the LD is used to drain CSF at 10 to $15 \mathrm{cc} /$ hr to facilitate healing of the dural closure by reducing hydrodynamic stress. For open, ASB craniotomy, watertight dural closure is achieved either by primary repair or with the use of a pedicled pericranial graft augmented with autologous fat and/or a synthetic polymer glue (DuraSeal, Covidian, Mansfield, Massachusetts, USA). For endoscopic procedures, autologous fat buttressed by Gelfoam (Baxter International, Inc., Deerfield, Illinois, USA) and DuraSeal is used. Significant defects in the floor of the ACF or high-flow CSF fistulae that may result from entry into the floor of the third ventricle are repaired with a vascularized nasoseptal flap augmented by the above mentioned closure materials. Nasal packs are used at times but are not relied upon for dural closure. Postoperatively, the $\mathrm{LD}$ is positioned at the level of the patient's shoulder and permitted to drain 10 to $15 \mathrm{cc} /$ hour in the neurosurgical intensive care unit under close monitoring. Patients remain flat while the drain is in place but are permitted to move around when the drain is clamped. Generally, drainage is performed for the first 3 days following surgery, after which the LD is clamped for 24 hours and, if there was no evidence of a CSF leak, the LD is removed. Postoperative CSF leaks are defined as any extraventricular extravasation of clear fluid such as rhinorrhea or a subgaleal collection.

The cumulative percentage of patients who experienced a postoperative CSF leak or other postoperative complication was calculated. Statistical analysis related to patient comorbidities was performed using Fischer's exact test $(p<0.05)$. Approval was obtained from the Loyola University Medical Center Institutional Review Board prior to initiating this study.

\section{Results}

Forty-three patients had LDs placed prior to elective ASB surgery. Surgical routes to the ASB included transnasal, transsphenoidal endoscopic approaches $(N=23)$, bifrontal craniotomy $(N=11)$, pterional craniotomy $(N=6)$ and cranio-orbito-zygomatic $(N=3)$ approaches.

Two patients with esthesioneuroblastomas had preoperative CSF rhinorrhea; in both instances, preoperative LD placement and closure with a nasoseptal flap augmented by synthetic polymer glue was effective in eliminating a postoperative CSF leak. Of the remaining 41 patients without signs or symptoms of a preoperative CSF leak who underwent LD placement prior to elective ASB tumor surgery, two patients developed postoperative CSF leaks $(2 / 41=4.9 \%$ 
Table 3 Postoperative complications following surgery involving lumbar drain placement

\begin{tabular}{|l|l|}
\hline Postoperative Complication & Incidence (\%) \\
\hline CSF leak & $2(4.7)$ \\
\hline Nausea/vomiting & $8(18.6 \%)$ \\
\hline Headache & $5(11.6)$ \\
\hline DVT & $2(4.7)$ \\
\hline STEMI & $1(2.3)$ \\
\hline
\end{tabular}

Abbreviations: CSF, cerebrospinal fluid; DVT, deep vein thrombosis; STEMI, ST segment elevation myocardial infarction.

overall postoperative CSF leak rate) that required a return to the operating room for definitive closure; both cases were in our endoscopic patient population $(N=21 ; 2 / 21=9.5 \%$ endoscopic postoperative CSF leak rate). One of the patients had a recurrent leak after the first postoperative repair and is described below in detail (Case report 1 ). No postoperative CSF leaks in the open ASB cohort were noted $(N=20)$. Other postoperative complications included headache, nausea, vomiting, deep vein thrombosis, and myocardial infarction (-Table 3): the most common complication noted was nausea $(N=8 ; 8 / 43=18.6 \%)$. We encountered no postoperative drain site hematomas, lower extremity weakness or pares- thesias, infections, or retained catheter tips on attempted removal.

Of the 41 patients without preoperative CSF leaks who had a LD placed prior to surgery, there were 20 males and 21 females. Although all patients had some degree of intracranial air on postoperative imaging, no instances of tension pneumocephalus were noted. Hypertension $(N=15)$, diabetes mellitus ( $N=5$ ), and the use of tobacco products (generally cigarette smoking; $N=22$ ) were confounding variables in $76 \%(n=31 / 41)$ of patients; no correlation was noted between these variables and the occurrence of a postoperative CSF leak. We did, however, encounter two significant postoperative complications in patients who had preoperative LD placement prior to craniotomy for ASB tumor resection. Those cases also merited separate discussion.

\section{Case Report \#1 (Recurrent CSF Rhinorrhea)}

This 73-year-old Caucasian woman with a history of diabetes mellitus, hypertension, and significant cardiac disease (for which she had previously undergone coronary artery bypass surgery) presented with headaches and blurry vision in her left eye. A bitemporal hemianopsia was noted on formal visual field testing. Magnetic resonance imaging (MRI) revealed a homogenously enhancing, sellar lesion with suprasellar extension and compression of the optic chiasm ( - Fig. 1). She
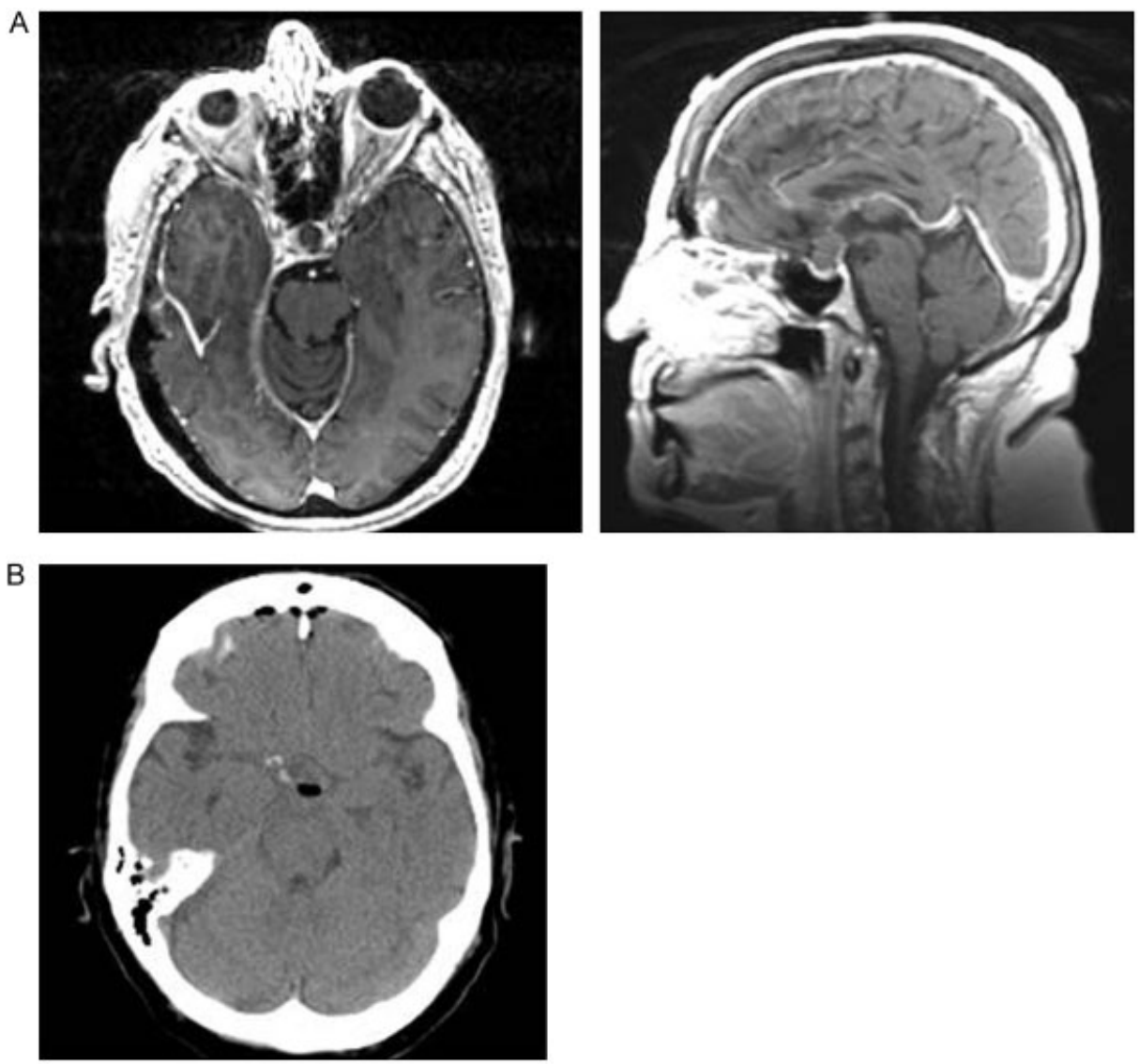

Fig. 1 Preoperative T1-weighted, gadolinium-enhanced axial and sagittal images (top) demonstrating an isointense, poorly enhancing sellar mass with suprasellar extension causing mass effect on the optic chiasm (A). Postoperative noncontrast computed tomography (CT) head with minimal pneumocephalus noted in the resection cavity and adjacent to the bilateral frontal lobes. No intracranial hemorrhage or evidence of edema (B). 

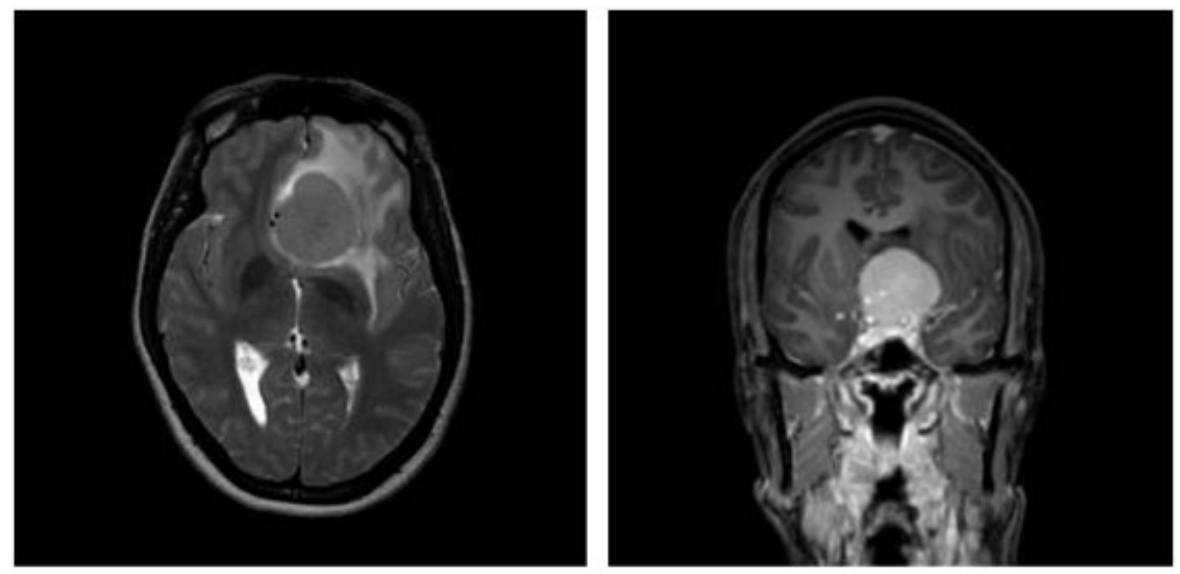

Fig. 2 Preoperative T2-weighted, noncontrast axial and coronal images revealing a well-circumscribed, homogenously contrast-enhancing, dural-based olfactory groove lesion measuring $3 \times 4 \mathrm{~cm}$ with evidence of peritumoral vasogenic edema.

had a LD placed prior to undergoing an uncomplicated endoscopic, transnasal, transsphenoidal resection of a benign pituitary adenoma with suprasellar extension and invasion of the floor of the third ventricle. At the conclusion of the case, the patient had a sellar floor reconstruction with abdominal fat bolstered by Gelfoam covered by DuraSeal with nasal packing. Postoperatively, the patient remained flat with the LD set at shoulder level set to drain 10 to $15 \mathrm{cc} / \mathrm{hr}$. Postoperative cranial computed tomography (CT) revealed minimal intracranial air and no evidence of hemorrhage (-Fig. 1). Postoperative polyuria and transient hypernatremia (peak Na $147 \mathrm{mmol} / \mathrm{L}$ ) required one dose of desmopressin (DDAVP; Sanofi-aventis, Bridgewater, New Jersey, USA). Approximately $300 \mathrm{cc}$ of CSF was drained via the LD over 2 days, the nasal packing was removed, and the LD was clamped and then removed on the fourth postoperative day with no evidence of a CSF leak.

On postoperative day 5 she had acute onset of nausea and a bout of emesis, after which brisk CSF rhinorrhea was noted. She was taken to the operating room for an endoscopic repair of the sella floor with abdominal fat graft and replacement of the LD. Following surgery, the LD was again set to drain at 10 to $15 \mathrm{cc} / \mathrm{hr}$. On the evening of postoperative day 2 , the patient acutely became hypotensive, requiring fluid resuscitation and intravenous phenylephrine (Neo-synephrine; Hospira, Inc., Lake Forest, Illinois, USA) to support her blood pressure. An echocardiogram revealed mild right heart strain, and a pulmonary embolism protocol CT revealed a linear filling defect in the right lower lobe. The LD was removed, an inferior vena cava filter placed, and a heparin drip initiated.

Two days later, CSF rhinorrhea was again noted. She was taken back to surgery and repeat endoscopy permitted visualization of the floor of the third ventricle and CSF leakage draining from the suprasellar cistern. This was repaired endoscopically with the placement of an abdominal fat graft and a nasoseptal vascularized flap. She returned to the neurological intensive care unit (ICU) with a third LD that drained between 275 and 350 cc of CSF daily until the drain was clamped on postoperative day 4 . The LD remained clamped through postoperative day 6 , at which time there were no signs or symptoms of a CSF leak, and the LD was removed. She was subsequently discharged home and has remained asymptomatic without evidence of a CSF leak.

\section{Case Report \#2 (Malignant Cerebral Edema)}

This 39-year-old otherwise healthy African American woman presented with a generalized tonic-clonic seizure and intermittent blurry vision. An MRI of the brain revealed a $3 \times 4 \mathrm{~cm}$ olfactory groove lesion suggestive of a meningioma (-Fig. 2). Other than a left superior nasal quadrantanopsia, she was neurologically intact. She was started on levetiracetam (Keppra; UCB Pharmaceuticals, Brussels, Belgium) and had no subsequent seizures.

She underwent a bifrontal craniotomy, cranialization of the frontal sinus, and a Simpson grade 3 resection of the tumor. Reconstruction of the dura of the floor of the ASB and sequestration of the frontal sinus from the intracranial space was performed using a pedicled, pericranial graft augmented by DuraSeal. Prior to surgery, an LD was inserted but kept clamped, and $10 \mathrm{mg}$ of dexamethasone (Decadron; Merck and Co., Inc., Whitehouse Station, New Jersey, USA), $1 \mathrm{~g}$ of Keppra, and $25 \mathrm{~g}$ of mannitol were administered intravenously (IV). Following dural opening, an additional $25 \mathrm{~g}$ of mannitol was administered IV and 10 cc of CSF was siphoned from the LD.

During the subfrontal/interhemispheric approach, the left frontal lobe was noted to be edematous and swollen. An additional $25 \mathrm{~g}$ of IV mannitol was given and another $10 \mathrm{cc}$ of CSF drained via the LD. Near-total surgical resection was achieved and a small portion of the tumor encasing the left anterior cerebral artery was left intentionally. Following surgery, the patient was extubated without incident and had a normal neurological examination. A postoperative cranial CT obtained within 6 hours of surgery showed an appropriate resection cavity without evidence of hemorrhage or ventricular dilatation. Mild pneumocephalus without tension was noted (-Fig. 3). In the neurosurgical ICU, the LD was leveled at the shoulder and set to drain $10 \mathrm{cc} / \mathrm{hr}$. Tumor 



Fig. 3 Immediate postoperative, noncontrasted computed tomography (CT) head with scant pneumocephalus and trace extra-axial hemorrhage (A). Left greater than right frontal hypodensity, representing persistent edema, continued mass effect, and reduced subfalcine herniation. Postoperative day 1, noncontrasted CT head with persistent bifrontal edema, worsened mass effect on the left lateral ventricle (B). Postoperative, noncontrasted CT head status postbifrontal decompressive craniectomy (C). Evidence of extensive cerebral edema, effacement of the sulcal-gyral pattern with loss of gray-white differentiation. Slit-like ventricles. Crowding of the basilar cisterns. Herniation through the craniectomy site.

pathology was consistent with a World Health Organization (WHO) II atypical meningioma with a $\mathrm{K}_{\mathrm{i}}-67$ proliferation index of $4 \%$ that was progesterone receptor positive and featured prominent nucleoli and several foci of necrosis.

Immediate postoperative diabetes insipidus and transient hypernatremia (peak Na $153 \mathrm{mmol} / \mathrm{L}$ ) required close fluid and electrolyte monitoring and one dose of IV DDAVP. She was also reported to be intermittently confused-oriented only to name and place-but the CSF drainage through the LD was continued as per the original protocol, draining $10 \mathrm{cc} / \mathrm{hr}$. Over the next 10 to 12 hours, she was reported to be awake and alert, with a nonfocal, normal neurological examination.

On the afternoon of postoperative day 1 , she had a brief episode of unresponsiveness and was noted to have sluggish, $6 \mathrm{~mm}$ dilated pupils bilaterally. The LD was immediately clamped and an emergent cranial CT scan revealed significant bifrontal cerebral edema with crowding of the basal cisterns (-Fig. 3). She was administered $100 \mathrm{~g}$ of IV mannitol, $20 \mathrm{mg}$ of IV Decadron, and $1 \mathrm{~g}$ of IV Keppra. The LD was discontinued. Electroencephalography (EEG) did not reveal any epileptiform discharges or subclinical seizures. Within an hour, the patient regained her normal neurological examination, an examination that she maintained until the evening of postoperative day 3-only requiring intermittent IV DDAVP to control elevated urine output.

On the evening of postoperative day 3 , the patient became acutely unresponsive. On examination, she was noted to have $6 \mathrm{~mm}$, nonreactive pupils with a dysconjugate gaze and flexor posturing. She was given $50 \mathrm{~g}$ of IV mannitol. She then proceeded to have a generalized, tonic-clonic (GTC) seizure which required administration of $4 \mathrm{mg}$ of IV lorazepam (Ativan, Baxter International, Inc., Deerfield, Illinois, USA) $1 \mathrm{~g}$ of IV Keppra, and $1 \mathrm{~g}$ of IV fosphenytoin (Cerebyx; Pfizer Inc., New York, New York, USA) before her tonic-clonic activity ceased. Subsequent cranial CT scan revealed persistent bifrontal cerebral edema with crowding of the basal cisterns. She gradually regained consciousness, had intermittent eye opening with spontaneous movement of all four extremities, and spontaneous, normal respirations, but she did not follow commands.

On the morning of postoperative day 4 , despite adequate antiepileptic medications, she had another GTC seizure, this time requiring intubation. The patient's cranial CT scan showed no change from the previous afternoon. A right frontal external ventricular drain (EVD) was placed with an opening pressure of $25 \mathrm{~cm}$ of $\mathrm{H}_{2} \mathrm{O}$ with an appropriate waveform. The drain was set to $10 \mathrm{~cm}$ above the pterion. After $20 \mathrm{cc}$ of CSF drained briskly, the catheter stopped draining, and the monitor showed a poor waveform. A leftsided intracranial pressure monitor was then placed and confirmed elevated pressures of between 21 and $24 \mathrm{~mm}$ Hg. The patient showed no signs of neurological improvement and was found to have fixed and dilated pupils, absent brain stem reflexes, and no movement of the extremities. She was taken to the operating room emergently for removal of her cranial bone flap and resection of swollen and infarcted brain tissue. Postoperatively, the patient did not recover any neurological function. A cranial CT scan showed progression of the already severe bifrontal edema with evidence of bilateral anterior cerebral artery infarctions (-Fig. 3). The next morning, EEG and cerebral blood flow studies confirmed brain death, and the family withdrew care.

\section{Case Report \#3 (Cerebral Edema)}

This 68-year-old Caucasian woman who had previously undergone radical hysterectomy and bilateral salpingo-oophorectomy for Stage II-A cervical carcinoma presented with a seizure prompting an MRI that demonstrated a $3 \times 3 \times 2 \mathrm{~cm}$ contrast-enhancing planum sphenoidale lesion suggestive of a meningioma (-Fig. 4). Prior to her scheduled admission for surgery, she developed pulmonary complaints and was diagnosed with a pulmonary embolus requiring a 6 -month course 

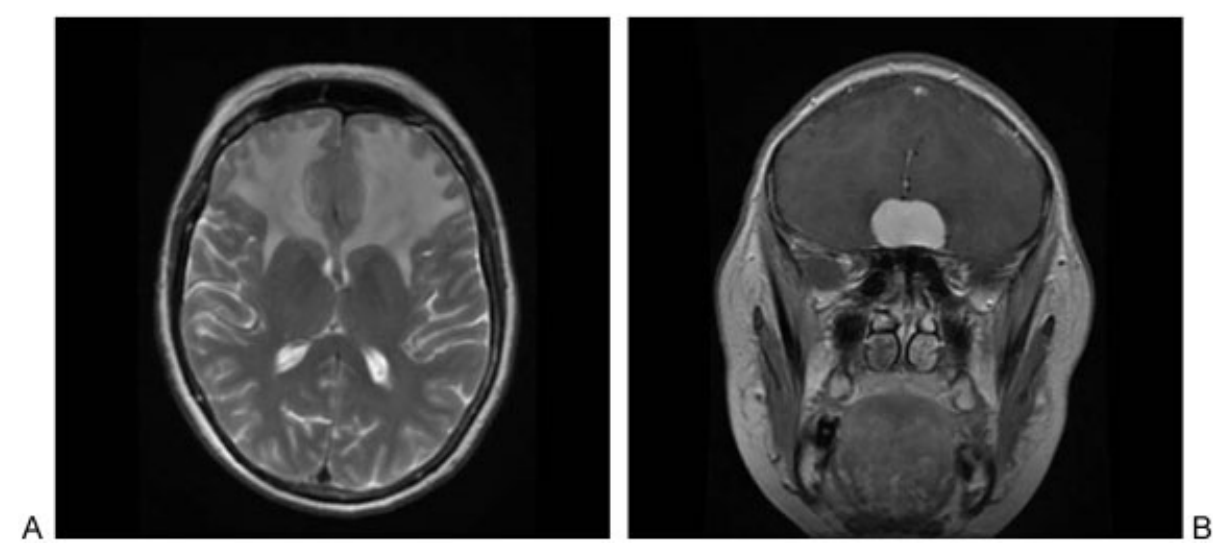

Fig. 4 Preoperative axial T2-weighted, noncontrasted enhanced (A) and coronal T1 gadolinium-enhanced (B) images showing a $3 \times 3 \mathrm{~cm}$, dural-based planum sphenoidale mass with local mass effect. Bifrontal vasogenic edema.

of warfarin (Coumadin; Bristol-Myers Squibb Company, New York, New York, USA). Otherwise, the patient was a nonsmoker with hypertension and hyperlipidemia.

With completion of warfarin therapy and normalization of coagulation parameters, she underwent an uncomplicated bifrontal craniotomy and gross total resection of the tumor that was found to be a WHO grade I meningioma without atypical features. At surgery, the frontal sinus was cranialized and then sequestered from the intracranial compartment by a pedicled pericranial graft that was also used to reconstruct the dural defect augmented by abdominal fat and DuraSeal. Prior to surgery, she received a loading dose $(15 \mathrm{mg} / \mathrm{kg}$ ) of IV phenytoin (Dilantin, Pfizer Inc., New York, New York, USA), in addition to her maintenance dose of Keppra and $10 \mathrm{mg}$ of IV Decadron. She received $25 \mathrm{~g}$ of IV mannitol just prior to dural opening. Following surgery, she was extubated uneventfully and had a normal neurological examination; postoperative CT scan revealed a complete tumor resection with minimal, nontension pneumocephalus (-Fig. 5).

On the morning of postoperative day 1 , the patient was neurologically intact, and her LD was opened to drain 10 to $15 \mathrm{cc} / \mathrm{hr}$. Through the afternoon and evening, she reported intermittent nausea and had several episodes of emesis. On postoperative day 2 , the patient became acutely somnolent with a labored breathing pattern requiring emergent intubation. The LD was clamped, and a cranial CT revealed bilateral frontal edema (-Fig. 5). She was administered $100 \mathrm{~g}$ of IV mannitol and $20 \mathrm{mg}$ of IV Decadron. Her neurological examination steadily improved, returning to normal and permitting successful extubation 12 hours later. She remained on a low dose of mannitol for 2 days and her Decadron was tapered over 1 week. The LD remained clamped. It was removed the next morning. Two subsequent cranial CT scans revealed resolving frontal edema (-Fig. 5). She was discharged 6 days after surgery with a normal neurological examination

A
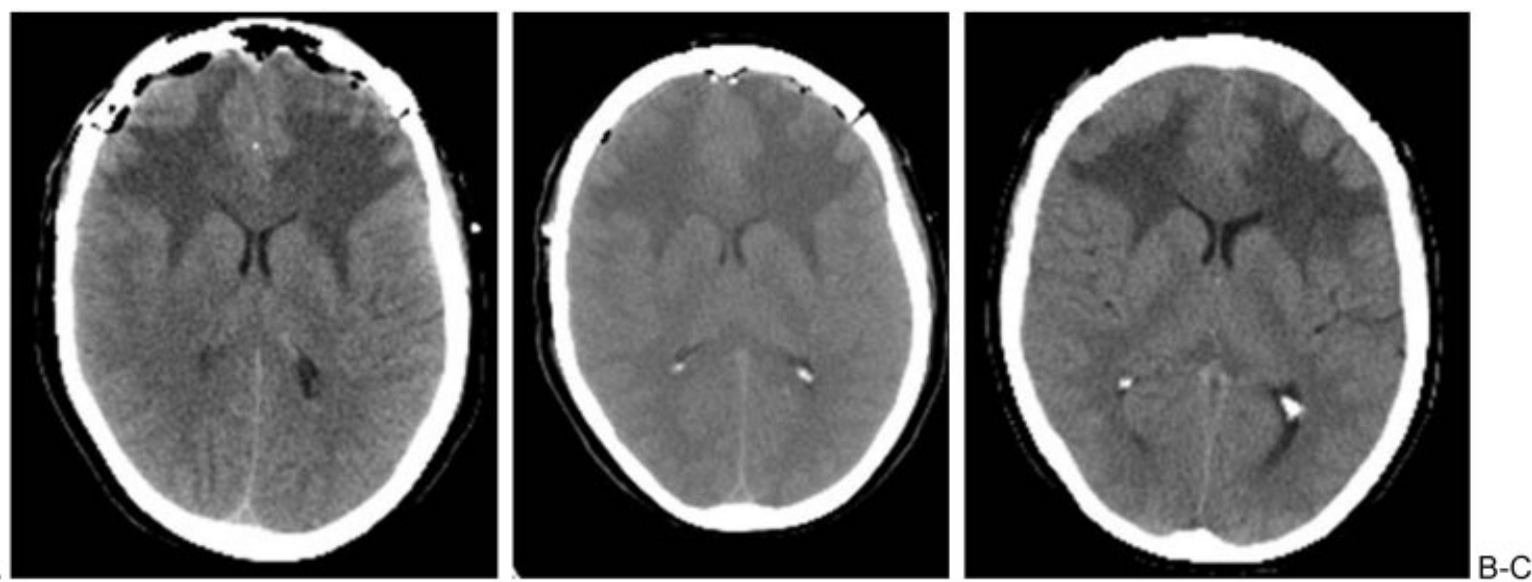

Fig. 5 Immediate postoperative, noncontrasted computed tomography $(C T)$ head with bifrontal hypodensity representing persistent cerebral edema (A). Local mass effect. Bifrontal pneumocephalus and scant extra-axial hemorrhage. Postoperative day 1 , noncontrasted CT head with persistent bifrontal edema with continued mass effect on the lateral ventricles (B). Improved pneumocephalus, extra-axial hemorrhage. Postoperative day 6 , noncontrasted CT head with resolving bifrontal cerebral edema, decreased mass effect, and more appropriately demarcated sulcal-gyral pattern (C). 
and continues to do well at follow up more than 12 months after surgery.

\section{Discussion}

A variety of surgical approaches to address pathology of the ASB are described and are effective. ${ }^{20-26}$ However, despite advances in technology and refinement of technique, postoperative CSF leaks are still observed in 5 to $20 \%$ of patients undergoing cranial base procedures. ${ }^{14,27-30}$ Inserting a drain into the lumbar subarachnoid cistern has become an acceptable strategy for mitigating postoperative CSF leak following cranial base procedures. ${ }^{2-16}$

In 2007, Bien et al performed a retrospective analysis of 150 posterior fossa craniotomy surgeries and noted that preoperative LD placement decreased the incidence of postoperative CSF leak by $23 \%{ }^{13}$ Similar practice is extrapolated to and has been found effective with ASB procedures. ${ }^{1}$ However, some authors question the utility of an LD altogether and others have challenged the prudence of routine, prophylactic LD placement prior to ASB surgery, describing an increased incidence of postoperative intracranial complication, in particular tension pneumocephalus. ${ }^{12,15}$ Some authors even present data suggesting that particular types of CSF leak, CSF rhinorrhea for example, are comparatively less likely to respond to LD placement. ${ }^{31}$

Weaver et al reported on 62 patients who underwent preoperative LD placement prior to thoracoabdominal aortic aneurysm repair. $^{32}$ The LD was inserted at the L2-3 level in $21 \%$, L3-4 level in 53\%, and L4-5 level in $25 \%$ of patients. Drains remained in place an average of 2.4 days. Two patients developed intradural hematomas at the insertion site; one of them had lower extremity neurological deficits as a result. No infectious or other complications as a result of the LD placement were reported. Yates et al did a retrospective review of 22 craniofacial procedures in 18 patients over a 54-month period and noted the development of pneumocephalus in 7 patients and tension pneumocephalus in 2 of these 7 patients. ${ }^{33}$ Both instances of tension pneumocephalus occurred in patients undergoing LD placement and was further complicated by the development of transient diabetes insipidus.

Ransom et al retrospectively evaluated 65 patients undergoing LD placement prior to endoscopic ASB surgery. ${ }^{19}$ The LD remained in place an average of 2 to 3 days. Four patients required repeat surgery for a postoperative CSF leak. Nine LD complications occurred in eight patients. A persistent lumbar CSF leak in five patients required epidural blood patch placement. Significant overdrainage, inadvertent disconnection, inappropriate opening, and a retained intradural catheter tip were noted each in one patient.

Roland et al retrospectively reviewed the charts of 32 patients undergoing LD placement for CSF fistula over a 3year period. ${ }^{10}$ Minor complaints included headache, nausea, and vomiting and were noted in 59\% of patients. Major complications were noted in $12.5 \%$ of patients and included unilateral occlusion of the posterior cerebral artery and unilateral vocal cord palsy. All complications, major and minor, were relieved by cessation of lumbar CSF drainage.
Pepper et al reported on data gathered from their population of 161 patients who underwent transglabelar, subcranial approaches to the ASB over a 14 -year period. ${ }^{15}$ Of these, 41 patients had an LD placed prior to surgery. They noted that routine placement of a LD was associated with an increased risk of tension pneumocephalus and intracranial complications and did not significantly reduce the incidence of CSF leak as a result of the operations.

Grady et al retrospectively reviewed the charts of 530 patients undergoing LD placement prior to transsphenoidal surgery. ${ }^{12}$ No new neurological deficits attributable to insertion of the LD were noted. Thirteen patients developed a spinal headache; seven of these required an epidural blood patch to relieve their symptoms. Their conclusion was that insertion of an LD in an anesthetized patient prior to surgery was a safe procedure.

This study revealed that $\mathrm{LD}$ insertion prior to surgery is an effective way to mitigate the risk of postoperative CSF leaks following open and endoscopic ASB procedures. The two cases of postoperative CSF leaks despite LD placement occurred in the endoscopic population and, in one instance, was easily remedied by a return to the operating room and careful reconstruction of the floor of the anterior cranial fossa.*

However, the patient with a recurrent CSF leak following initial repair merits careful analysis. As noted in this patient, in cases where the floor of the third ventricle is violated, a high-flow CSF fistula results that may not be eliminated by autologous fat and synthetic polymer glue packing even when augmented by a LD. Such instances and recurrent CSF rhinorrhea cases require a vascularized nasoseptal flap closure of the ASB augmented by autologous fat, Gelfoam, and synthetic glue. As Kassam et al noted, and as done in this case, the use of a vascularized nasoseptal flap is a significant factor in achieving a successful closure of the CSF fistula. ${ }^{1}$

The second and third case vignettes highlight the problem of cerebral edema that accompanies some tumors such as meningiomas, particularly atypical or anaplastic meningiomas, and the risk of lumbar CSF drainage in these patients following open ASB procedures. One patient had a WHO grade II atypical meningioma, whereas the other patient had a WHO grade I meningioma but with significant vasogenic edema, as is sometimes noted with the secretory variant of the tumor. Similar problems of cerebral edema may be observed in Kadish stage $C$ esthesioneuroblastomas that invade the dural and pial boundaries. Cerebral edema may exist prior to surgery in these patients and may be exacerbated by surgery, particularly if venous structures are

\footnotetext{
The anterior cranial fossa was accessed via the endoscopic, transnasal approach. An AlloDerm (LifeCell Corp., The Woodlands, Texas, USA) graft was bolstered with abdominal fat and an additional layer of thick AlloDerm used to cover the entire skull base dural defect. The AlloDerm matrix was draped into the frontal sinus along the anterior face of the posterior table and laterally along the orbits with the posterior edge tucked into the clival recess. DuraSeal and Avitene (Davol, Inc., Cranston, Rhode Island, USA) further augmented the skull base repair, and two Merocel (Medtronic, Inc., Mystic, Connecticut, USA) sponges packed the bilateral nares.
} 
sacrificed to get access to the tumor. Lumbar CSF drainage in these cases can result in significant neurological compromise due to downward herniation syndromes either through the tentorial incisura or the foramen magnum. In Patient \#2, the CT obtained after neurological decline did indeed demonstrate crowding of the cerebellar tonsils into the foramen magnum that was not previously noted. Both patients responded to IV mannitol boluses. Patient \#2 also developed problems with seizures, which are frequently seen with atypical or anaplastic meningiomas. Her neurological status declined almost 48 hours after the LD was removed. Although this decline could be attributed to seizures and cerebral edema, it is also possible that the LD played a role. Bloch and Regli described a case of a patient who developed brainstem and cerebellar dysfunction 3 days after removal of a LD that was placed following a pterional craniotomy for clipping of an aneurysm. ${ }^{34}$ An MRI scan demonstrated both tentorial and foramen magnum herniation. The patient was successfully treated with ventricular drainage, an epidural blood patch, and being placed in a Trendelenburg position. Presumably, a persistent CSF fistula existed even after the LD was removed, allowing CSF egress through the drain site, and some element of postoperative cerebral edema or swelling existed that led to the herniation syndrome described.

As with any nonrandomized, retrospective review without a control arm, there are limitations to this study. In addition, the three cases described may represent anecdotal evidence at best. However, the lessons are germane to the practice of all practicing skull base surgeons and consistent with the experience of other surgeons. It has also caused us to alter our practice, and this has resulted in excellent outcomes with no further LD-induced complications. At present, we insert a LD only in endoscopic cases with suprasellar or limited intracranial tumor extension without mass effect. We do not insert a LD for large, intracranial ASB lesions with significant mass effect or vasogenic edema. Drainage of CSF is only commenced after a 12-hour observation period in the neurosurgical ICU with a CT scan that demonstrates no evidence of cerebral edema, tension pneumocephalus, or hydrocephalus and with open basal cisterns. Drainage is performed at 10 to $15 \mathrm{cc} /$ hour during daytime hours from 8:00 Am to 5:00 PM with close neurological monitoring for 3 days. The drain is clamped on the fourth day and then, after 24 hours, removed in the absence of a CSF leak.

\section{Conclusion}

Our experience demonstrates that LD placement is generally well-tolerated by patients-we had no postoperative complications directly attributable to drain placement-and is an effective means by which to minimize postoperative CSF leak after ASB craniotomy. Recurrent or high-flow CSF leaks require a vascularized pedicled nasoseptal flap reconstruction. The risk of brain herniation syndromes with LD placement is present in patients with large intracranial tumors, especially those prone to generating significant vasogenic edema, and LD placement is avoided in these patients. Neurological decline in a patient with an LD in place necessitates an immediate IV bolus of mannitol and a cranial CT to rule out tension pneumocephalus or intracranial hypertension. Neurological decline may be observed even after drain removal and requires similar management.

\section{References}

1 Kassam AB, Prevedello DM, Carrau RL, et al. Endoscopic endonasal skull base surgery: analysis of complications in the authors' initial 800 patients. J Neurosurg 2011;114:1544-1568

2 Magliulo G, Sepe C, Varacalli S, Fusconi M. Cerebrospinal fluid leak management following cerebellopontine angle surgery. J Otolaryngol 1998;27:258-262

3 Fishman AJ, Hoffman RA, Roland JT Jr, Lebowitz RA, Cohen NL. Cerebrospinal fluid drainage in the management of CSF leak following acoustic neuroma surgery. Laryngoscope 1996;106:1002-1004

4 Fishman AJ, Marrinan MS, Golfinos JG, Cohen NL, Roland JT Jr. Prevention and management of cerebrospinal fluid leak following vestibular schwannoma surgery. Laryngoscope 2004;114:501-505

5 Hoffman RA. Cerebrospinal fluid leak following acoustic neuroma removal. Laryngoscope 1994;104(1 Pt 1):40-58

6 Nutik SL, Korol HW. Cerebrospinal fluid leak after acoustic neuroma surgery. Surg Neurol 1995;43:553-556, discussion 556-557

7 Leonetti J, Anderson D, Marzo S, Moynihan G. Cerebrospinal fluid fistula after transtemporal skull base surgery. Otolaryngol Head Neck Surg 2001;124:511-514

8 Bryce GE, Nedzelski JM, Rowed DW, Rappaport JM. Cerebrospinal fluid leaks and meningitis in acoustic neuroma surgery. Otolaryngol Head Neck Surg 1991;104:81-87

9 Becker SS, Jackler RK, Pitts LH. Cerebrospinal fluid leak after acoustic neuroma surgery: a comparison of the translabyrinthine, middle fossa, and retrosigmoid approaches. Otol Neurotol 2003;24:107-112

10 Roland PS, Marple BF, Meyerhoff WL, Mickey B. Complications of lumbar spinal fluid drainage. Otolaryngol Head Neck Surg 1992;107:564-569

11 Açikbaş SC, Akyüz M, Kazan S, Tuncer R. Complications of closed continuous lumbar drainage of cerebrospinal fluid. Acta Neurochir (Wien) 2002;144:475-480

12 Grady RE, Horlocker TT, Brown RD, Maxson PM, Schroeder DR; Mayo Perioperative Outcomes Group. Neurologic complications after placement of cerebrospinal fluid drainage catheters and needles in anesthetized patients: implications for regional anesthesia. Anesth Analg 1999;88:388-392

13 Bien AG, Bowdino B, Moore G, Leibrock L. Utilization of preoperative cerebrospinal fluid drain in skull base surgery. Skull Base 2007;17:133-139

14 Deschler DG, Gutin PH, Mamelak AN, McDermott MW, Kaplan MJ. Complications of anterior skull base surgery. Skull Base Surg 1996;6:113-118

15 Pepper JP, Lin EM, Sullivan SE, Marentette LJ. Perioperative lumbar drain placement: an independent predictor of tension pneumocephalus and intracranial complications following anterior skull base surgery. Laryngoscope 2011;121:468-473

16 Allen KP, Isaacson B, Purcell P, Kutz JW Jr, Roland PS. Lumbar subarachnoid drainage in cerebrospinal fluid leaks after lateral skull base surgery. Otol Neurotol 2011;32:1522-1524

17 Lee TJ, Huang CC, Chuang CC, Huang SF. Transnasal endoscopic repair of cerebrospinal fluid rhinorrhea and skull base defect: tenyear experience. Laryngoscope 2004;114:1475-1481

18 Casiano RR, Jassir D. Endoscopic cerebrospinal fluid rhinorrhea repair: is a lumbar drain necessary? Otolaryngol Head Neck Surg 1999;121:745-750

19 Ransom ER, Palmer JN, Kennedy DW, Chiu AG. Assessing risk/ benefit of lumbar drain use for endoscopic skull-base surgery. Int Forum Allergy Rhinol 2011;1:173-177

20 Sekhar LN, Nanda A, Sen CN, Snyderman CN, Janecka IP. The extended frontal approach to tumors of the anterior, middle, and posterior skull base. J Neurosurg 1992;76:198-206 
21 Raveh J, Turk JB, Lädrach K, et al. Extended anterior subcranial approach for skull base tumors: long-term results. J Neurosurg 1995;82:1002-1010

22 Kaptain GJ, Vincent DA, Sheehan JP, Laws ER Jr. Transsphenoidal approaches for the extracapsular resection of midline suprasellar and anterior cranial base lesions. Neurosurgery 2001;49:94-100, discussion 100-101

23 Jho HD, Ha HG. Endoscopic endonasal skull base surgery: Part 1The midline anterior fossa skull base. Minim Invasive Neurosurg 2004; $47: 1-8$

24 Chandler JP, Pelzer HJ, Bendok BB, Hunt Batjer H, Salehi SA. Advances in surgical management of malignancies of the cranial base: the extended transbasal approach. J Neurooncol 2005;73: $145-152$

25 Dusick JR, Esposito F, Kelly DF, et al. The extended direct endonasal transsphenoidal approach for nonadenomatous suprasellar tumors. J Neurosurg 2005;102:832-841

26 Frank G, Pasquini E, Doglietto F, et al. The endoscopic extended transsphenoidal approach for craniopharyngiomas. Neurosurgery 2006;59(1, Suppl 1):ONS75-ONS83, discussion ONS75-ONS83

27 Batra PS, Luong A, Kanowitz SJ, et al. Outcomes of minimally invasive endoscopic resection of anterior skull base neoplasms. Laryngoscope 2010;120:9-16
28 Kaptain GJ, Kanter AS, Hamilton DK, Laws ER. Management and implications of intraoperative cerebrospinal fluid leak in transnasoseptal transsphenoidal microsurgery. Neurosurgery 2011;68 (1, Suppl Operative):144-150, discussion 150-151

29 Harvey RJ, Nogueira JF, Schlosser RJ, Patel SJ, Vellutini E, Stamm AC. Closure of large skull base defects after endoscopic transnasal craniotomy. Clinical article. J Neurosurg 2009;111: 371-379

30 Laws ER, Kanter AS, Jane JA Jr, Dumont AS. Extended transsphenoidal approach. J Neurosurg 2005;102:825-827, discussion 827828

31 Mangus BD, Rivas A, Yoo MJ, et al. Management of cerebrospinal fluid leaks after vestibular schwannoma surgery. Otol Neurotol 2011;32:1525-1529

32 Weaver KD, Wiseman DB, Farber M, Ewend MG, Marston W, Keagy BA. Complications of lumbar drainage after thoracoabdominal aortic aneurysm repair. J Vasc Surg 2001;34:623-627

33 Yates $\mathrm{H}$, Hamill M, Borel CO, Toung TJ. Incidence and perioperative management of tension pneumocephalus following craniofacial resection. J Neurosurg Anesthesiol 1994;6:15-20

34 Bloch J, Regli L. Brain stem and cerebellar dysfunction after lumbar spinal fluid drainage: case report. J Neurol Neurosurg Psychiatry 2003;74:992-994 